Ann. Biol. anim. Bioch. Biophys., 1978, 18 (3), 645-650

\title{
Une méthode d'extraction quantitative des phospholipides totaux dans les aliments, les digesta et les fèces
}

\author{
par A. THEWIS, E. FRANÇOIS *, Marie-France THIELEMANS * \\ avec la collaboration technique de F. DUPONT *
}

Chaire de Physiologie animale ef de Zootechnie,

Faculté des Sciences agronomiques de l'Ełat,

* Station de Chimie ef de Physique agricoles, Centre de Recherches agronomiques de l'Etat, 5.800 Gembloux, Belgique.

Summary. Extraction of fotal phospholipids in feeds, digesta and feces. A quantitative method.

This report presents a method for the quantitative extraction of phospholipids in feeds, feces and digesta. Successive extraction of the samples were carried our first with warm solvents : ethanol, chloroform and chloroform-methanol mixture $(2: 1 \mathrm{v} / \mathrm{v})$, then with a cold chloroform-methanol-nitric acid mixture (645:322:33 v/v/v). Phospholipid levels were estimated from total lipid phosphorus contents; determination was complete and reproducible. It was necessary to carry out at least 2 determinations for each sample, owing to the risk of phosphate contamination of organic $P$.

\section{Infroduction.}

L'extraction est l'une des étapes les plus critiques du dosage des lipides en général et des phospholipides en particulier dans des échantillons de végétaux, de contenus gastro-intestinaux et de fèces. En effet, ils s'y trouvent à l'état libre, engagés dans des structures membranaires complexes, liés à des protéines ou encore sous forme de combinaisons insolubles, des savons principalement.

Divers systèmes de solvants ont été utilisés pour extraire les phospholipides de matériaux de nature diverse. Citons les systèmes chloroforme : méthanol : eau $(3: 2:$ $0,17 \mathrm{v} / \mathrm{v}$ ) puis chloroforme : méthanol : acide nitrique concentré (2:1:0,01 v/v) préconisés par Zhukov ef Vereshchagin (1976) pour extraire les lipides polaires des graines de soja ; l'éthanol, le chloroforme et le chloroforme : méthanol (2:1 v/v) utilisés successivement à chaud sur des échantillons d'herbes et de jus de rumen par Dawson et Hemington (1974) ; le diméthoxyméthane : méthanol (4:1 v/v) employé par Boucrot (1972) pour extraire les lipides des contenus gastro-intestinaux de rats; le chloroforme : méthanol $(2: 1 \mathrm{v} / \mathrm{v})$ acidifié par l'acide chlorhydrique concentré $(2 \mathrm{p} .100 \mathrm{v} / \mathrm{v})$ utilisé par Sklan ef al. (1973) pour extraire les lipides dans les con tenus intestinaux de poulets; et enfin, le mélange méthanol : dichlorure d'éthylène (1 : 
3 v/v) utilisé par Ali, Kuksis et Beveridge (1966) pour extraire les phospholipides de fèces humaines préalablement acidifiées à l'aide d'acide chlorhydrique $4 \mathrm{~N}$.

Parmi ces différents auteurs, seuls Zhukov et Vereshchagin (1976) prêtent une attention toute particulière à l'aspect exhaustif de l'extraction des lipides polaires des graines de soja. Malheureusement, la méthode proposée exige un appareillage spécial et ne se prête guère aux dosages en série. De plus, aucun auteur, à notre connaissance, n'a évalué le risque de contamination du phosphore phospholipidique par le phosphore non lipidique. Elle n'est évidemment à craindre que si on dose les phospholipides à partir de leur teneur en phosphore.

En conséquence, au cours de nos travaux portant sur la sécrétion ef l'absorption des composés phosphorés dans le tube digestif du mouton (Thewis, François ef Thielemans, à paraître), nous avons été amenés à élaborer une technique satisfaisante de dosage des phospholipides dans des échantillons d'aliments, de contenus gastrointestinaux et de fèces de ruminants.

La méthode présentée est une combinaison des techniques préconisées par Dawson ef Hemington (1974) ef Zhukov ef Vereshchagin (1976). La purification des extraits est, quant à elle, inspirée de la technique de Folch, Lees et Stanley (1957).

\section{Matériel et méthodes.}

\section{1. - Extraction des phospholipides.}

Les extractions sont réalisées sur le matériel frais ou éventuellement lyophilisé. Dans tous les cas, on s'assure du bon contact de la matière à extraire avec les solsolvants; éventuellement, on réduit l'échantillon en fines particules soit au moyen d'un ultra-turrax soit par simple broyage.

Tous les réactifs utilisés sont de qualité « Pour analyse ».

Un poids exactement connu de matière $(5 \mathrm{à} 10 \mathrm{~g})$ est additionné de $30 \mathrm{ml}$ d'éthanol absolu et chauffé au bain-marie à $80^{\circ} \mathrm{C}$ pendant $15 \mathrm{mn} .60 \mathrm{ml}$ de chloroforme sont ensuite ajoutés à l'alcool. Ce mélange est placé au bain-marie à $50^{\circ} \mathrm{C}$ pendant $30 \mathrm{mn}$ et agité de temps à autre. On filtre sur entonnoir de Büchner ${ }^{1}$ en s'aidant du vide de manière à recueillir quantitativement le filtrat directement dans une ampoule à décanter. Le résidu solide lavé plusieurs fois au chloroforme est récupéré quantitativement et de nouveau extrait, cette fois, par $50 \mathrm{ml}$ de chloroforme-méthanol $(2: 1 \mathrm{v} / \mathrm{v})$ au bain-marie à $50^{\circ} \mathrm{C}$ pendant $30 \mathrm{mn}$. L'extrait est filtré comme décrit ci-dessus et ajouté au premier, dans l'ampoule à décanter.

Cette première phase s'inspire de la méthode décrite par Dawson et Hemington (1974). On peut ainsi traiter une douzaine d'échantillons par jour.

Le résidu solide subit ensuife deux extractions supplémentaires avec respectivement 100 et $50 \mathrm{ml}$ de chloroforme-méthanol-acide nitrique concentré (645:322 : $33 \mathrm{v} / \mathrm{v} / \mathrm{v}$ ). La première extraction dure une nuit à $2-3^{\circ} \mathrm{C}$, la seconde, $3 \mathrm{~h}$. Après chaque macération, on filtre sous vide, on rince au chloroforme-méthanol-acide nitrique ; les deux extraits sont alors combinés dans une seconde ampoule à décanter.

(1) Filtre Schleicher et Schull $n^{0} 589^{1}$. 
Tous les extraits sont ensuite lavés ef purifiés par agitation en présence de 0,2 volume d'une solution aqueuse de $\mathrm{NaCl}(9 \mathrm{~g} / 1)$ (Folch, Lees ef Stanley, 1957). On laisse alors décanter pendant une nuit.

La phase inférieure contient le phosphore phospholipidique et la supérieure, le phosphore non lipidique. Chaque phase est recueillie quantitativement dans un ballon à long col rodé de $250 \mathrm{ml}$. La phase inférieure est décantée lentement ; la phase supérieure est aspirée sous vide directement dans un ballon à col rodé, en vue de la minéralisation. Les parois de l'ampoule sont rincées deux ou trois fois à l'aide de $10 \mathrm{ml}$ de la solution de Folch, Lees et Stanley (1957) ${ }^{1}$; ils sont ensuite aspirés dans le ballon contenant la phase supérieure. Puis on rince l'ampoule à l'aide d'environ $10 \mathrm{ml}$ d'un mélange ayant une composition proche de celle de la phase inférieure (pure solvent lower phase, Folch, Lees et Stanley, 1957) ; on les récupère dans le ballon contenant la couche inférieure. Les phases sont évaporées à sec à l'évaporateur rotatif sous vide, à $45^{\circ} \mathrm{C}$ pour la couche inférieure, à $60^{\circ} \mathrm{C}$ pour la supérieure. Le résidu est alors minéralisé à l'aide de $25 \mathrm{ml}$ de mélange nitro-perchlorique $(3: 1 \mathrm{v} / \mathrm{v})$ dans le même ballon auquel on adapte un appareil de Chat (1966). Ce dispositif à reflux comprenant un piège pour les vapeurs acides, permet de travailler dans d'excellentes conditions de sécurité et de rapidité. Il est conseillé de laisser agir le mélange oxydant à froid pendant une nuit avant de minéraliser.

\section{2. - Dosage du phosphore.}

Le phosphore est dosé par colorimétrie. On utilise le complexe phosphovanadomolybdate (Misson, 1908) pour les concentrations supérieures à 15 ppm; pour les concentrations plus faibles, on adopte la méthode de Fogg et Wilkinson (1958), dans laquelle le complexe phosphomolybdate est réduit par l'acide ascorbique. L'automatisation des dosages permet d'obtenir une excellente précision.

3. - Vérification du caractère exhaustif de l'extraction et de la reproductibilité de la méthode.

Des extractions sont effectuées en double selon le mode opératoire décrit cidessus sur des échantillons d'aliment, de fèces ef de contenus gastro-intestinaux prélevés à différents endroits du tube digestif. Le résidu solide récupéré après les deux extractions au chloroforme-méthanol-acide nitrique en subit une troisième à l'aide de $50 \mathrm{ml}$ de ce mélange pendant $2 \mathrm{~h}$. L'extrait est ensuite traité comme décrit pécédemment.

Par ailleurs, des extraits des couches inférieures, provenant d'échantillons menés parallèlement aux précédents, font l'objet d'une analyse par chromatographie sur couche mince (gel de silice 60 , plaque de $0,25 \mathrm{~m} / \mathrm{m}$ ). Les systèmes de solvants utilisés sont d'une part : éther de pétrole : éther éthylique : acide acétique (90:10:1), d'autre part : éther de pétrole : éther éthylique : acide formique $(60: 40: 1,5)$. Les vapeurs d'iode sont utilisées comme révélateur.

(1) $\mathrm{CHCl}_{3}=30 \mathrm{ml} ; \mathrm{CH}_{3} \mathrm{OH}=480 \mathrm{ml} ; \mathrm{H}_{2} \mathrm{O}$ distillée $=464 \mathrm{ml} ; \mathrm{CaCl}_{2} \cdot 2 \mathrm{H}_{2} \mathrm{O}-6,1 \mathrm{p} .100=8,5 \mathrm{ml}$. 


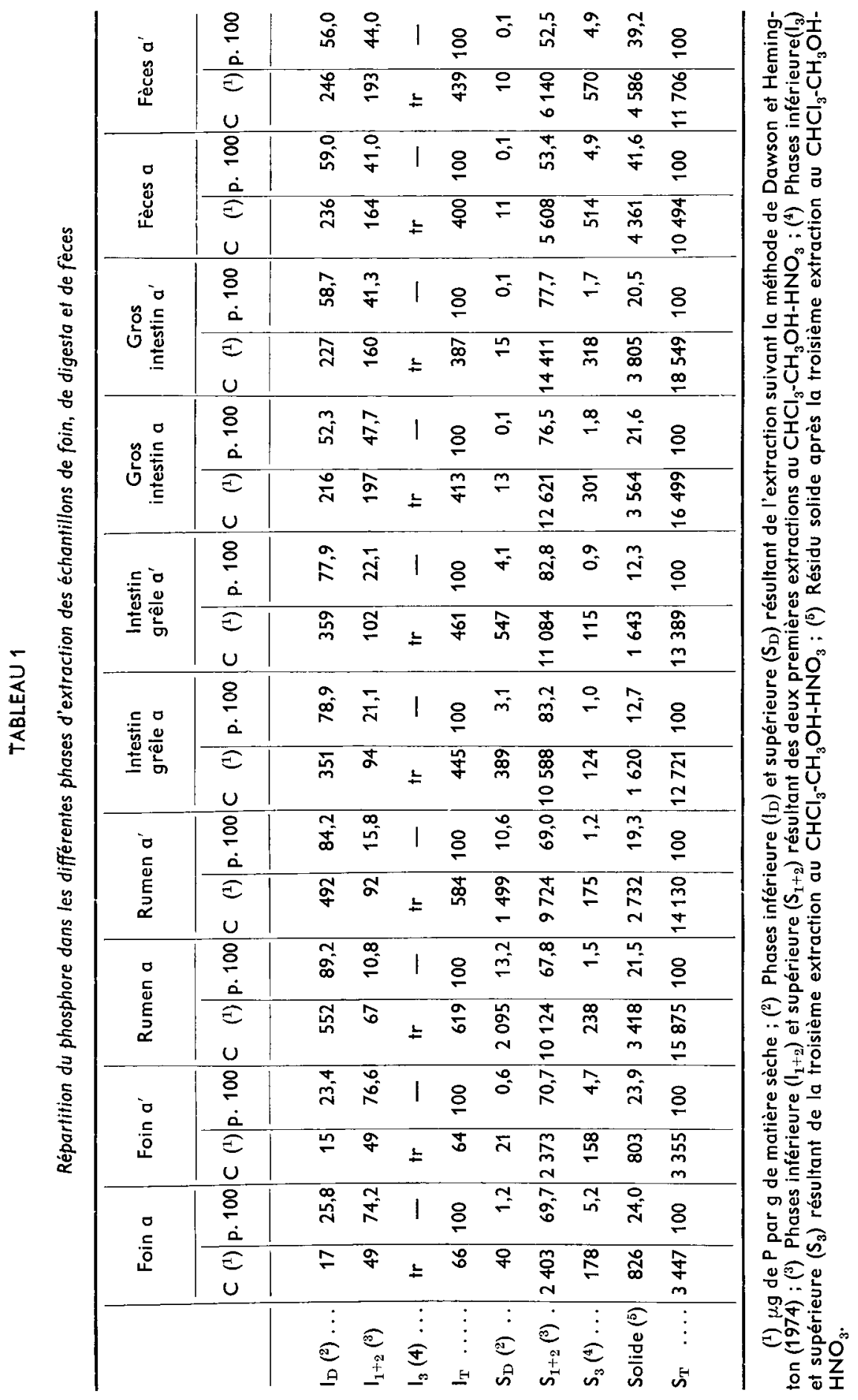


4. - Evaluation de la contamination du P d'origine phospholipidique par le P d'origine minérale.

Afin d'évaluer l'importance de la contamination éventuelle du $\mathrm{P}$ phospholipidique par le $\mathrm{P}$ non lipidique, une dose connue de ${ }^{32} \mathrm{P}$, sous forme d'orthophosphate de sodium, est ajoutée à 88 extraits avant lavage et séparation des 2 phases. Ces 2 opérations sont effectuées comme décrit ci-dessus.

Après évaporation, les phases sont minéralisées. Dans la solution obtenue après minéralisation, on dose le radiophosphore par évaporation d'un poids connu de celleci sur coupelle en polystyrène. Le détecteur employé est du type à courant gazeux, à fenêtre mince et à faible mouvement propre (1 CPM) ${ }^{1}$.

\section{Résultats et discussion.}

L'extraction des phospholipides doit d'après Zhukov ef Vereshchagin (1976) être réalisée en deux étapes : dans des conditions relativement douces d'abord, plus sévères ensuite, notamment si on veut éviter la destruction de certains composés lipidiques très fragiles.

Comme on le constate au tableau 1, le mélange chloroforme : méthanol : acide nitrique permet, suivant la nature de l'échantillon, d'extraire 10 à 75 p. 100 du phosphore phospholipidique.

L'acidification des échantillons ou des solvants par les acides nitrique ou chlorhydrique est d'ailleurs recommandée par Ali, Kuksis et Beveridge (1966), Sklan et al. (1973) et par Zhukov et Vereshchagin (1976). Selon ces derniers auteurs, il se pourrait qu'en milieu acide, les liaisons ioniques entre les lipides et certains constituants du résidu, tels que les ions alcalino-terreux, soient rompues. Par ailleurs, au niveau des matières fécales, l'acidification permet la destruction des complexes insolubles calcium-phosphates-acides gras et autres savons calciques présents à ce niveau (Ali et Kuksis, 1967).

Si nous avons ainsi montré la nécessité d'acidifier les solvants pour améliorer l'extraction des phospholipides dans nos échantillons, la présence de traces de $\mathrm{P}$

TABLEAU 2

Evaluation du risque de contamination du phosphore lipidique par le phosphore non lipidique

\begin{tabular}{|c|c|c|}
\hline $\begin{array}{l}\text { Niveau de contamination } \\
\qquad(*)\end{array}$ & Nombre de cas observés & en p. 100 \\
\hline 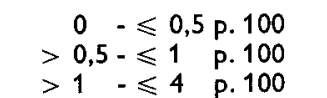 & $\begin{array}{l}43 \\
31 \\
14\end{array}$ & $\begin{array}{l}48,9 \\
35,2 \\
15,9\end{array}$ \\
\hline Total & 88 & 100,0 \\
\hline
\end{tabular}

(*) Pourcentage d'activité retrouvée dans les couches inférieures.

(1) Nuclear Chicago, Searle. 
uniquement dans les extraits $I_{3}$ montre que deux extractions au chloroforme : méthanol : acide nitrique suffisent. L'examen des extraits $I_{3}$ en chromatographie sur couche mince confirme d'ailleurs l'absence de phospholipides à ce niveau.

Les quantités de phosphore extraites dans $\mathrm{I}_{1+2}$ sont extrêmement faibles comparativement à celles contenues dans $S_{1+2}$. II s'ensuit que les valeurs de $\mathrm{I}_{1+2}$ sont très sensibles à une contamination, toujours possible comme on le constate au tableau 2.

En ce qui concerne la reproductibilité des dosages, il semble que l'on doive tolérer des écarts de 6 à 7 p. 100 pour deux analyses d'un même échantillon. Ce résultat nous paraît très acceptable pour des niveaux de phosphore aussi faibles. La bonne reproductibilité des résultats traduit par ailleurs indirectement la validité de la technique ainsi que l'aspect quantitatif malgré les nombreuses manipulations.

En conclusion, la méthode que nous proposons est exhaustive et quantitative. Les éventuelles contaminations seront aisément décelées si on prend la précaution de faire les extractions en double.

Reçu en août 1977.

Accepté en janvier 1978.

\section{Références}

ALI S. S., KUKSIS A., 1967. Excretion of phospholipids by men on fat-free diet. Can. J. Biochem., 45 , $689-702$

ALI S. S., KUKSIS A., BEVERIDGE J. M. R., 1966. Excretion of bile acids by three men on a fat-free diet. Can. J. Biochem., 44, 957-969.

CHAT G., 1966. Nouvelle méthode de minéralisation des végétaux pour les analyses chimiques. C. R. Acad. Agric. Fr., 51, 1087-1093.

BOUCROT P., 1972. Is there an entero-hepatic circulation of the bile phospholipids ? Lipids, 7, 282288.

DAWSON R. M. C., HEMINGTON N., 1974. Digestion of grass lipids and pigments in the sheep rumen. Br. J. Nutr., 32, 327-340.

FOGG D. N., WILKINSON N. T., 1958. The colorimetric determination of phosphorus. Analyst, 83, 406-414.

FOLCH J., LEES M., STANLEY G. H. S., 1957. A simple method for the isolation and purification of total lipids from animal tissues. J. biol. Chem., 226, 497-509.

MISSON G., 1908. Kolorimetrische Phosphorbestimmung im Stahl. Chemikerzeitung, 32, 633.

SKLAN D., BUDOWSKI P., ASCARELLI I., HURWITZ S., 1973. Lipid absorption and secretion in the chick : effect of raw soybean meal. J. Nutr., 103, 1299-1305.

THEWIS A., FRANÇOIS E., THIELEMANS M.-F. Etude quantitative de l'absorption et de la sécrétion du phosphore total et d'origine phospholipidique dans le tube digestif du mouton (à paraître).

WATHELET J. P., SEVERIN M., IMPENS R., 1972. Etude des lipides de Morchella rofunda Pers. Analyse des acides gras. Bull. Rech. agron. Gembloux, 7, 350-357.

ZHUKOV A. V., VERESHCHAGIN A. G., 1976. Quantitative content of total polar lipids in soybean seeds. J. om. Oil Chem. Soc., 53, 1-7. 